\title{
Endoscopic Assessment of Colorectal Cancer with Superficial or Deep Submucosal Invasion Using Magnifying Colonoscopy
}

\author{
Shinji Tanaka', Nana Hayashi', Shiro Oka ${ }^{1}$ and Kazuaki Chayama ${ }^{2}$ \\ Departments of ${ }^{1}$ Endoscopy and ${ }^{2}$ Gastroenterology and Metabolism, Hiroshima University Hospital, Hiroshima, Japan
}

\begin{abstract}
Among early colorectal carcinoma, endoscopic treatment is generally indicative for cases with intramucosal to submucosal (SM) superficial invasion, because cases with SM deep invasion should be treated surgically due to the risk of lymph node metastasis. It is important, therefore, to distinguish between superficial and deep SM invasion in early colorectal carcinoma prior to treatment. In this review we assessed the clinical usefulness and knack of pit pattern and narrow band imaging (NBI) diagnosis using magnifying observation. $\mathrm{V}_{\mathrm{N}}$ type pit pattern, type C3 in NBI Hiroshima classification and NBI type 3 in NBI international colorectal endoscopic (NICE) classification are useful predictors of SM deep invasion. In NBI magnifying observation evaluation of both the vascular pattern and surface pattern are important. We have to use pit pattern diagnosis and NBI magnifying diagnosis as the situation demands with the knowledge of both advantage and disadvantage in each diagnostic method.
\end{abstract}

Key Words: Colorectal subumucosal carcinoma; Invasion depth; Pit pattern; Narrow band imaging; Magnifying colonoscopy

\section{INTRODUCTION}

The progress in endoscopy, from forceps biopsy to pit pattern-based diagnosis using magnifying observation and optical diagnosis using image-enhanced endoscopy such as narrow band imaging (NBI), has led the changes in the clinical and pathological diagnosis of colorectal tumors. At present, endoscopic therapy is going to be adopted for final complete excisional biopsy. ${ }^{1}$

In early colorectal carcinoma, intramucosal carcinoma does not metastasize but lymph node metastasis occurs in approximately $10 \%$ of submucosal (SM) carcinoma cases. Therefore, care must be exercised in the clinical treatment of SM carcinoma. In Japan, the Japanese Society for Cancer of the Colon and Rectum guidelines 2010 for the treatment of colorectal cancer ${ }^{2}$ identify the following lymph node metastasis risk factors for SM colorectal carcinoma that has been completely re-

\footnotetext{
Received: February 13, 2013 Revised: March 8, 2013

Accepted: March 8, 2013

Correspondence: Shinji Tanaka

Department of Endoscopy, Hiroshima University Hospital, 1-2-3 Kasumi, Minami-ku, Hiroshima 734-8551, Japan

Tel: +81-257-5538, Fax: +81-253-2930, E-mail: colon@hiroshima-u.ac.jp

(c) This is an Open Access article distributed under the terms of the Creative Commons Attribution Non-Commercial License (http://creativecommons.org/ licenses/by-nc/3.0) which permits unrestricted non-commercial use, distribution, and reproduction in any medium, provided the original work is properly cited.
}

sected endoscopically (Fig. 1): 1) SM invasion depth of more than $1,000 \mu \mathrm{m} ; 2$ ) positive vascular invasion; 3 ) poorly differentiated adenocarcinoma, signet ring cell carcinoma, or mucinous carcinoma; 4) high grade tumor budding at the SM invasive part.

If the factors indicated in the guidelines are detected in an endoscopic resection specimen, additional lymph node dissection surgery should be considered. Of these pathological factors, only SM invasion depth can be determined before treatment. The other factors are diagnosed during pathological examination after the resection of SM colorectal carcinoma. Nevertheless, it is meaningless to perform endoscopic therapy for apparent colorectal carcinoma with deep SM invasion, because surgical resection must be performed after endoscopic therapy. Moreover, incomplete resection of a lesion prevents its precise pathological diagnosis, and the remnant local lesion may induce metastasis. ${ }^{3}$

Thus, clinical diagnosis before treatment is extremely important for early colorectal carcinoma with superficial or deep SM invasion. In this review, we focused on pit pattern and NBI diagnosis using magnifying colonoscopy and summarized the present situation and future perspectives of optical colonoscopic diagnosis. 


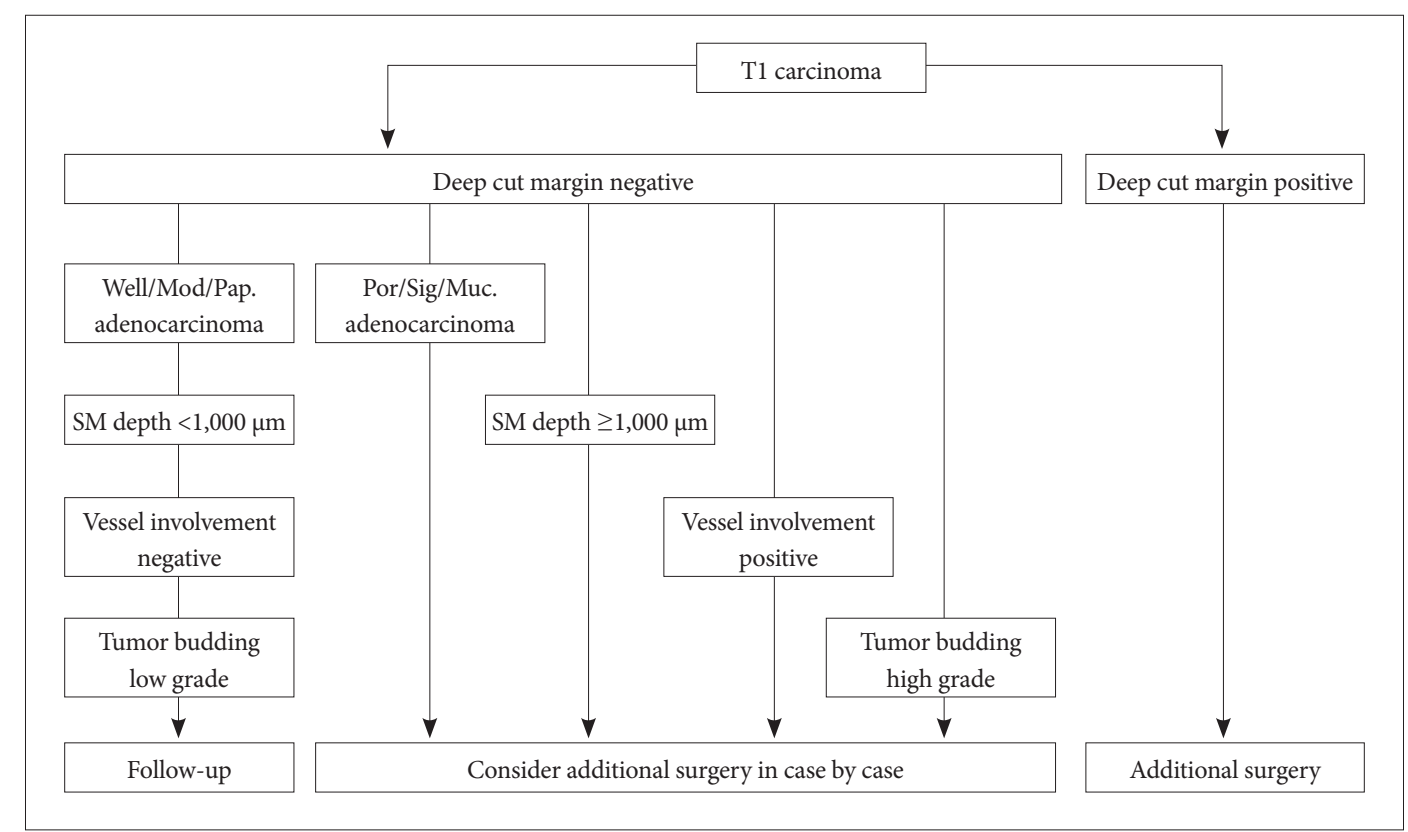

Fig. 1. Therapeutic strategy for colorectal submucosal (SM) carcinoma resected endoscopically (Japanese guideline for colorectal cancer treatment, 2010).

\section{PIT PATTERN DIAGNOSIS}

Clinical introduction of magnifying endoscopic observation of the large intestine is largely attributable to the establishment of pit pattern diagnosis by Kudo et al. ${ }^{4}$ and the development of a magnifying video colonoscope that is capable of facilitating an in vivo diagnosis. Currently, the magnifying video colonoscope is widely used, not only in Japan, but also in many countries around the world. The insertion ability, operationality, and specifications of high-resolution magnifying video colonoscopes, which are now commercially available, are similar to those of ordinary video colonoscope without magnifying function. Magnifying observation can be easily performed by optical zoom switch to enhance ordinary observation. At present, high-resolution magnifying video colonoscopes are used in general clinical examinations and treatment of the alimentary tract. ${ }^{5}$

\section{Pit pattern classification of colorectal tumors}

The pit, or crypt opening (CO), pattern of colorectal tumors is generally categorized according to the Kudo and Tsuruta classification systems (Fig. 2). ${ }^{5}$ The type I pit pattern indicates nonneoplastic mucosa. The type II pit pattern indicates hyperplastic polyp or superficial type serrated adenoma and sessile serrated adenoma/polyp (SSA/P). The type- $\mathrm{III}_{\mathrm{L}}$ pit pattern primarily indicates a protruded, nodule aggregated type lesion, or an adenoma with mild to moderate atypia. The type $\mathrm{III}_{\mathrm{s}}$ pit pattern is characteristic of a depressed lesion and primarily indicates tubular adenomas. The type IV pit pattern is observed in large protruded, nodule aggregated type lesions, which represent tubulovillous adenoma and mucosal carcinoma in many cases. The type $\mathrm{V}$ pit pattern is subclassified into type $V_{I}$, tumors with an irregular glandular structure, and type $V_{N}$, tumors with a clear amorphous area. ${ }^{5}$ Type $V_{I}$ is an index for adenoma with severe atypia or SM carcinoma. Type- $\mathrm{V}_{\mathrm{N}}$ is an index for carcinoma with deep SM invasion. Pit pattern diagnosis using this classification can reveal the histological grade and invasion depth of lesions.

Pit pattern classification is primarily based on histogenesis and the development of differentiated type adenocarcinoma, i.e., from adenoma-carcinoma sequence of adenoma to lowgrade differentiated-type adenocarcinoma to high-grade differentiated type adenocarcinoma. Magnifying observation of a $\mathrm{CO}$ with a magnifying colonoscope facilitates the evaluation of the structural atypia of the lesion's superficial tissue, the crypt destruction and its extent, and the existence of desmoplastic reaction. Magnifying observation does not reveal the structure of the deep region of a lesion. However, on the basis of unexplained close correlation between 1) atypia and the invasion depth of a tumor and 2) the structural atypia of the tumor surface (pit pattern) in the process of tumor development in the normal mucosa to invasive growth to deep SM invasive carcinoma, qualitative diagnostic modalities have been developed using pit pattern findings.

If the latest high-resolution video colonoscope is used, a large pit structure can be roughly diagnosed on close observation without magnification. However, a small or irregular pit pattern cannot be diagnosed correctly without magnifying 


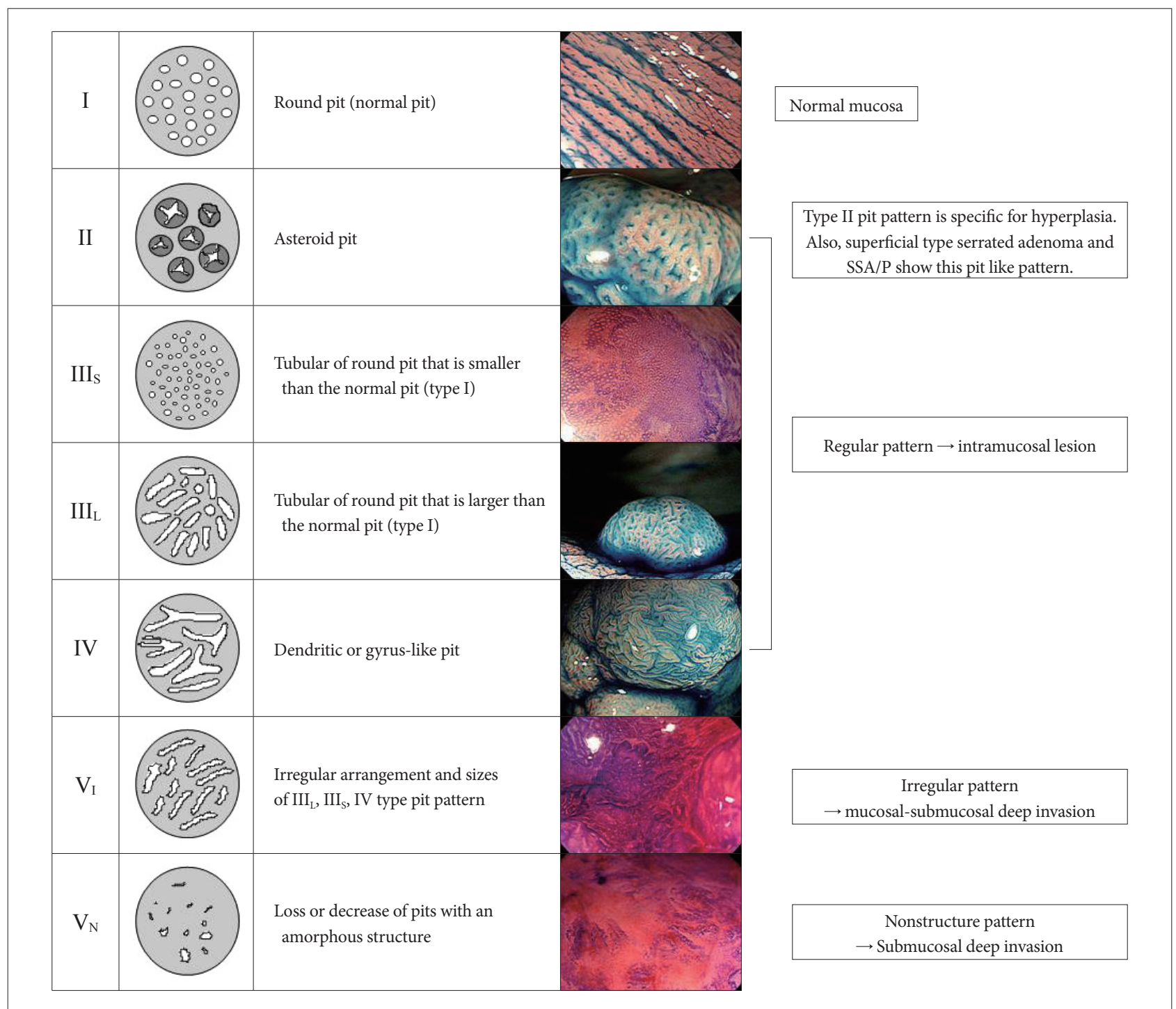

Fig. 2. Pit pattern classification of colorectal neoplasia (Adapted from Tanaka et al. Gastrointest Endosc 2006;64:604-613, with permission from Elsevier).

observation using indigo carmine dye spraying or crystal violet staining.

Recently, a pit pattern of undifferentiated type early colorectal carcinoma was diagnosed and reported, but only a small number of such cases have been reported. ${ }^{6}$ Diagnostic modalities for undifferentiated type early colorectal carcinoma using pit pattern findings have not yet been established.

An increasing number of cases regarding the relationship between pit pattern diagnosis of hyperplasia like lesions and the serrated pathway, which has recently attracted attention as a new carcinogenic pathway for SSA/P and serrated adenoma, has been noted. ${ }^{7}$ In Japan, the histological diagnostic criteria for SSA/P are currently being defined. ${ }^{8}$ During the early stages of tumorigenesis, histological changes begin at the lower part of a crypt. Because no abnormality can be observed on the surface of the lesion, pit pattern diagnosis based on magnifying observation, which can be used to diagnose structural atypia of the tumor surface, cannot be used to detect tumorigenesis during the early stages. Diagnostic modalities using pit pattern findings are being developed and research on the diagnostic modalities is ongoing.

\section{Subclassification of type $V_{I}$ pit pattern}

Qualitative diagnosis of lesions exhibiting the type $V_{\text {I }}$ pit pattern is not accurate because of the existence of various histological grades and invasion depths. Therefore, type $V_{I}$ pit pattern is classified as highly and slightly irregular pit patterns (Figs. 3, 4). The highly irregular $\mathrm{V}_{\mathrm{I}}$ pit pattern is defined as that in which the existing pit pattern is damaged or destroyed.

Possible findings to cite for highly irregular $\mathrm{V}_{\mathrm{I}}$ pit patterns 
Tanaka S et al.

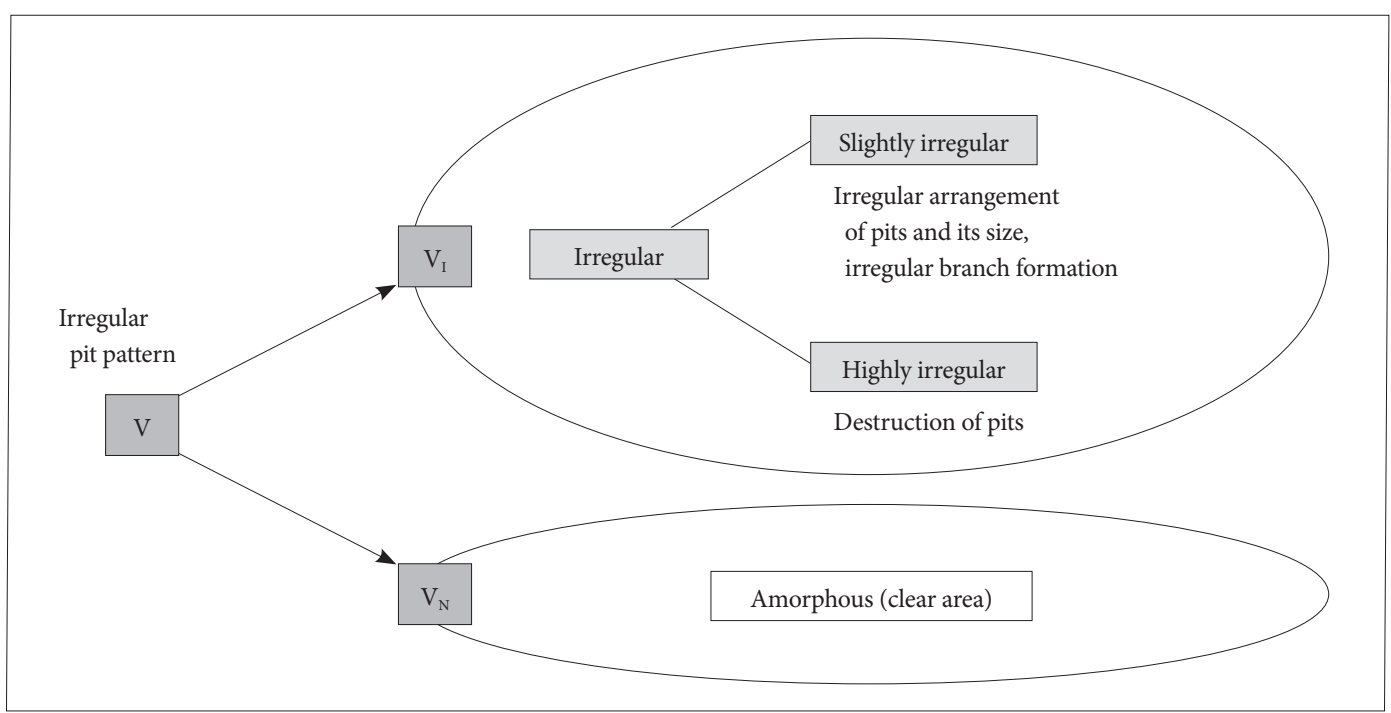

Fig. 3. Hakone agreement regarding the subclassification of type- $V$ pit patterns (April 2004, Hakone). In April 2004, a consensus meeting was held in Hakone and the definitions of findings of type- $V$ pit pattern-based diagnosis were unified. The type- $V$ pit pattern was subclassified into type- $\mathrm{V}_{\mathrm{I}}$, which represents an irregular glandular structure, and type- $\mathrm{V}_{\mathrm{N}}$, which represents a clear amorphous region. The invasive pattern, highly irregular glands, and the scratch sign were included as indices for submucosal carcinomas. Of note, colorectal tumors with a clear amorphous region were defined as type- $\mathrm{V}_{\mathrm{N}}$.
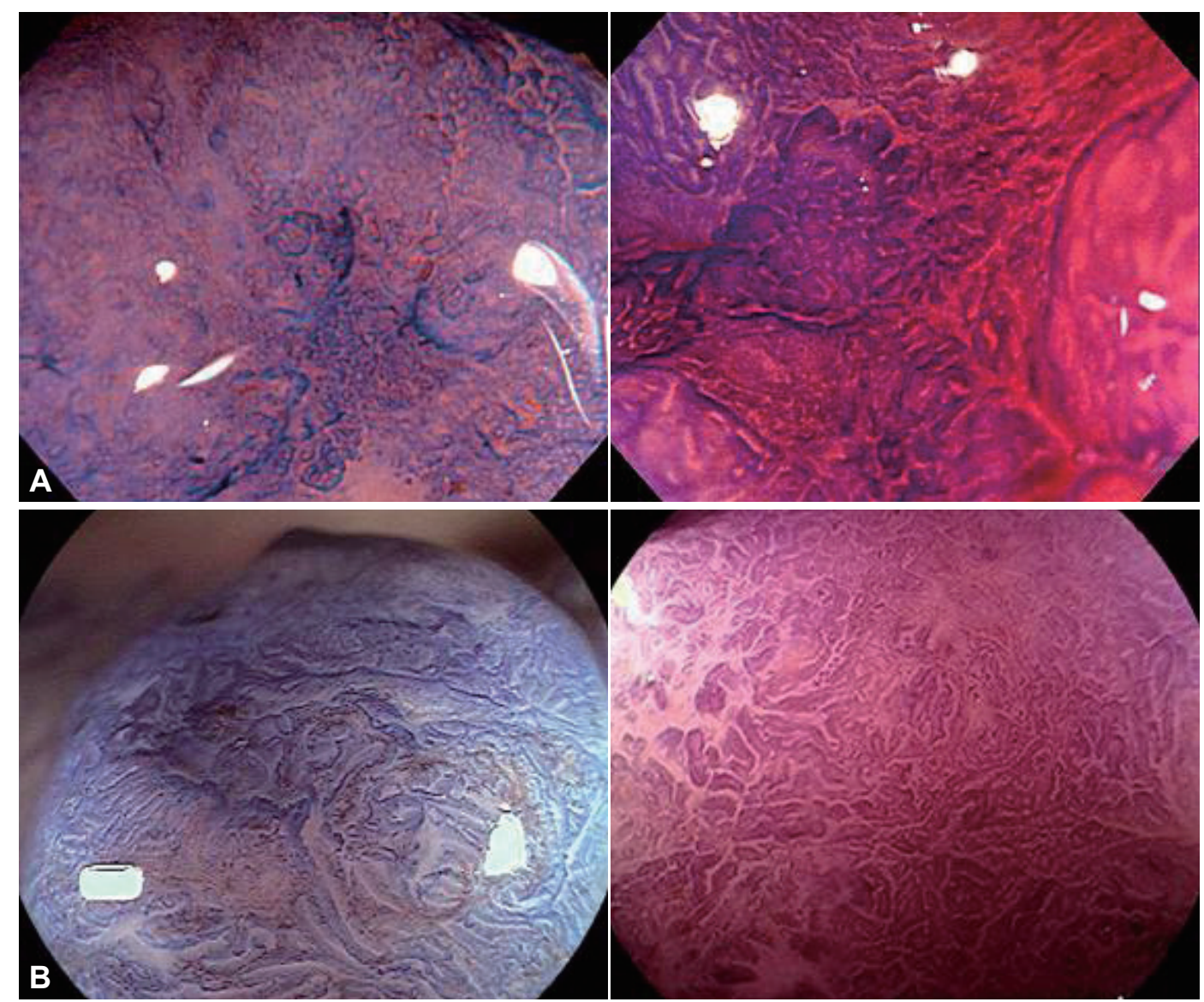

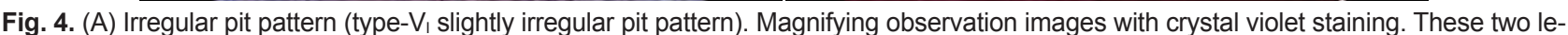
sions can be classified as the type- $V_{\text {, }}$ pit pattern with slight irregularity. No amorphous region is observed, but irregular pit structures such

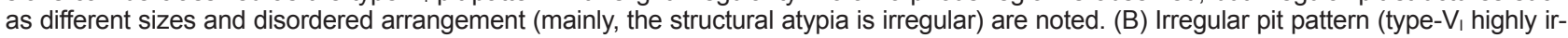
regular pit pattern). Magnifying observation images with crystal violet staining. These two lesions can be classified as the type- $V_{1}$ pit pattern with high irregularity. No clear amorphous region is observed, but pit structures with an irregular, rough margin; a decrease in stainability in the stromal area (surface pattern); and an unclear pit margin (the pit opening is damaged or destroyed) are noted. 
Table 1. $V_{1}$ Type Pit Pattern Subclassification and Histology/Invasion Depth in Adenoma and Early Carcinoma

\begin{tabular}{cccccc}
\hline \multirow{2}{*}{$\mathrm{V}_{\mathrm{I}}$ subtype } & No. of cases & \multicolumn{4}{c}{ Histology/Invasion depth } \\
\cline { 3 - 6 } & & Adenoma & $\mathrm{M}$ & $\mathrm{SM}<1,000 \mu \mathrm{m}$ & $1,000 \mu \mathrm{m} \leq \mathrm{SM}$ \\
\hline Slightly irregular & $44(100)$ & $15(34)$ & $21(48)$ & $3(7)^{\mathrm{a})}$ & $5(11)$ \\
Highly irregular & $41(100)$ & $2(5)$ & $7(17)$ & $12(29)^{\mathrm{a})}$ & $20(49)$ \\
\hline
\end{tabular}

Values are presented as number (\%).

${ }^{\text {a) }} p<0.01$.

include irregular margins, unclear contours, narrowed lumens of the pit, decreased and disappeared stainability in the stromal area, and scratch sign. However, methods for using these findings have not been unified; for example, there is no consensus as to which finding is essential for diagnosis of the highly irregular $V_{I}$ pit pattern or how many findings are required to establish the diagnosis. Therefore, the diagnostic criteria for the highly irregular $V_{\text {I }}$ pit pattern differ slightly across institutes. Table 1 shows data on adenoma and early carcinoma obtained at Hiroshima University. The diagnostic rate for lesions exhibiting the highly irregular $\mathrm{V}_{\mathrm{I}}$ pit pattern with an SM invasion depth of more than $1,000 \mu \mathrm{m}$ was approximately $50 \% .{ }^{9}$ For lesions diagnosed as exhibiting the slightly irregular $\mathrm{V}_{\mathrm{I}}$ pit pattern, those with an SM invasion depth of less than 1,000 $\mu \mathrm{m}$ accounted for approximately $90 \%$ of all lesions. Therefore, the slightly irregular $\mathrm{V}_{\mathrm{I}}$ pit pattern was considered for use as a diagnostic index for endoscopic treatment applicable lesions. ${ }^{9}$ In an institute, the diagnostic rate for lesions exhibiting the highly irregular $\mathrm{V}_{\mathrm{I}}$ pit pattern with an SM invasion depth of more than $1,000 \mu \mathrm{m}$ was $80 \%$ to $90 \%$. The difference in the diagnostic rate is attributable to the fact that methods of using the findings for the highly irregular $V_{\text {I }}$ pit pattern have not yet been unified.

\section{NBI}

\section{Principles of NBI}

Epithelial tumors in the alimentary tract develop and grow in the mucosal epithelium. For efficiently detecting epithelial tumors in their early stages, an endoscope that can examine fine structures such as microvascular architecture and pit pattern in the mucosal surface layer with high sensitivity should be used. In order to create this type of endoscope, an NBI system based on the wavelength dependence of light was developed. ${ }^{10-12}$ In general, the depth of light penetration into the biological tissue strongly depends on the wavelength of light. In other words, the penetration depth of short-wavelength light into the biological tissue is shallow, it is diffused and absorbed near the tissue surface, and the light is observed as reflected light. In contrast, long-wavelength light can be propagated deep into the tissue. Therefore, to adjust the sensitivity of an endoscope to a change in the fine structure in the mucosal surface layer, the sensitivity characteristics of the endoscope must be shifted to the short-wavelength side. In the NBI system, the band of an optical filter in a frame-sequential type video endoscope is narrowed, the band is shifted to the shortwavelength side, and the penetration depth of the observation light is localized to the mucosal surface layer. After these adjustments, the NBI system can visualize the microvascular architecture in the mucosal surface layer with high sensitivity. Also, NBI shows a structure enhancement effect on the lesion surface.

Thus, NBI enables diagnosis by facilitating the detailed visualization of fine structures and microvessels in the mucosal surface layer. For correct diagnosis using NBI, comprehensive evaluation of both the fine structures and microvessels is important.

\section{Basic findings of NBI magnifying endoscopic observation}

\section{Vascular patterns}

In NBI magnifying observation of an adenomatous lesion, the microvessels between the pits in the outermost layer of the mucosa are dark brown and exhibit a regular capillary mesh network. For carcinomas, heterogeneous vascular diameter, irregular blood flow, and disordered blood vessel distribution occur along with infiltration, proliferation, inflammatory cell infiltration, and desmoplastic reaction..$^{13}$ By using NBI magnifying observation to analyze microvessel visibility and diameter, distribution heterogeneity, and irregularity of vessels, it is possible to differentiate colorectal lesions as tumor/nontumor and adenoma/carcinoma. On the basis of the background provided above, many institutes have proposed various classifications and evaluation methods. ${ }^{14-16}$ However, many problems and limitations are associated with performing qualitative tumor diagnosis using only vascular patterns that can be visually identified using NBI magnifying observation. ${ }^{13}$

\section{Surface patterns}

In NBI magnifying observation of an adenomatous lesion, the microvessels between the pits in the outermost layer of the mucosa are dark brown and exhibit a regular capillary mesh network. However, a "pit-like" mucosal region reveals 
nothing on observation (no blood vessels observed). By employing the ability of NBI to enhance structure, the "pit-like" region can be indirectly diagnosed. ${ }^{13,15,17}$ For carcinomas, heterogeneous vascular diameter, irregular blood flow, disordered microvessel distribution, and destruction of the pit-like structures and interfoveolar mucosa all co-occur along with cancerous cell infiltration, proliferation, inflammatory cell infiltration, and desmoplastic reaction. ${ }^{18}$ The "pit-like structure" recognized in NBI magnifying observation is a structure in which an actual $\mathrm{CO}$ and the marginal crypt epithelium are combined. ${ }^{17}$ The fine surface pattern has been assigned various nomenclatures such as the pit-like structure and the white zone. In the Consensus Symposium at the 79th Annual Meeting of the Japan Gastroenterological Endoscopy Society (May 2010), these nomenclatures for the fine surface pattern were unified and replaced with "surface pattern" in colorectal tumors. $^{17}$

\section{NBI magnifying observation-based classification of colorectal tumors}

NBI magnifying observation-based classifications for colorectal tumors, which have been reported, are summarized below.

\section{(1) Sano classification}

The Sano classification considers the microvascular architecture alone. In this classification, the capillary pattern (CP) is defined as a meshed brown capillary arrangement surrounding the pit orifice. On the basis of its visibility, caliber variation, tortuosity, and stoppage, the CP is classified as type I, II, IIIA, or IIIB. CP type I corresponds to a pattern of normal or hyperplastic polyps that precludes visual identification of microvessels. CP type II corresponds to a pattern in which microvessels of uniform diameter appear to be surrounding the pit orifice. CP type IIIA is observed in carcinomas. CP type IIIB is observed in deep SM invasive carcinomas. ${ }^{14}$

\section{(2) Hiroshima classification ${ }^{15}$}

The Hiroshima classification comprehensively considers both surface pattern and microvascular architecture. In this classification, type A represents normal or color-faded colorectal lesions with invisible microvessels (an index for hyperplastic lesions). Type B corresponds to colorectal tumors in which a regular surface pattern or a regular meshed microvessel network pattern surrounding a pit can be observed (an index mainly for adenomas). Type $\mathrm{C}$ accounts for colorectal tumors with an irregular surface pattern or without specific structure-this type is sub-classified into types C1 to C3. Type C3 includes colorectal tumors in which the surface pattern disappears, an avascular area appears, and irregular blood vessels or fractured microvessels are interspersed (an index for deep SM invasive carcinomas) (Fig. 5). Although an irregular surface pattern is observed in types $\mathrm{C} 1$ and $\mathrm{C} 2$, these types differ with regard to heterogeneity in vascular diameter and distribution. Table 2 shows the relationship between the Hiroshima classification and pathological findings in colorectal hyperplasia, adenoma and early carcinoma.

\section{(3) Showa classification}

The Showa classification considers the pit-like structure and microvascular architecture and the morphological expression of these characteristics. This classification differs slightly from the two category-based classification systems described above because colorectal tumors are classified according to the microvessel pattern surrounding the pit. In colorectal tumors with a faint pattern, the microvessels surrounding the pit are undertint and difficult to identify visually. In tubular adenomas with a network pattern, the microvessels with a uniform diameter form an elliptical network around the pit. In villous and tubulovillous adenoma with a dense pattern, the microvessels are thick and dense and the epithelium is thickly congested. In a deep SM invasive carcinoma with an irregular pattern, the microvessels exhibit caliber variation, high tortuosity, stoppage-inducing continuity, and little deterioration. For a deep SM invasive carcinoma with a sparse pattern, the microvessels often observed in the recess become sparse. ${ }^{16}$

\section{(4) NBI International Colorectal Endoscopic (NICE)}

classification as an international classification system ${ }^{17,19}$

As mentioned above, NBI magnifying endoscopic observation for colorectal tumors has been classified and evaluated in various ways. ${ }^{14-17}$ The classification must be simple and easy to understand; otherwise, it cannot be widely used. However, if the classification is too simple and includes only a definition, it cannot be used for the accurate diagnosis of colorectal tumors.

When closely observing a colorectal tumor using the latest high-resolution video colonoscope, fine structures such as the pit structure on the surface are visible to some degree without the use of magnifying endoscopic observation. The use of NBI enhances structural visualization, and hence, the vascular pattern on a tumor can be observed in addition to the surface pattern. In Western countries, the magnifying endoscope has not been widely used in clinical practice until now. Even in Japan, the magnifying endoscope has not been sufficiently used to examine the colorectal neoplasia. On the basis of the background information provided above, a simple system was proposed to categorically classify NBI findings for use in close observations with a high-resolution electronic endoscope. The NICE classification ${ }^{17,19}$ was proposed by the Colon 

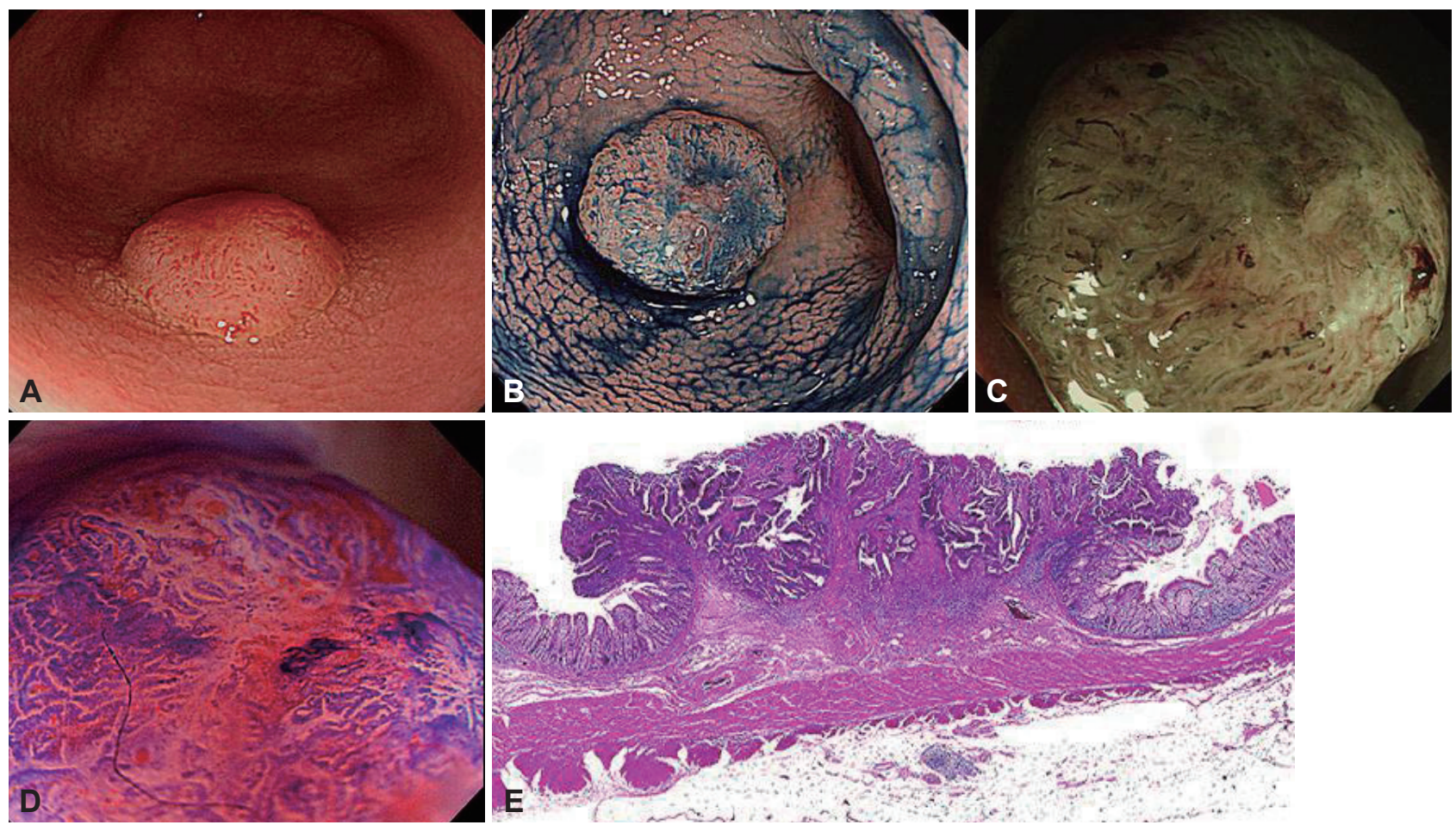

Fig. 5. A case of colorectal carcinoma with submucosal deep invasion. (A) Sessile polypoid lesion in standard colonoscopic view. (B) Indigo carmine dye spraying view. (C) Narrow band imaging magnifying view. Surface pattern is unclear to amorphous and there is an avascular area with scattered vessel (Hiroshima classification type C3). (D) Magnifying view with crystal violet staining. Center of the lesion shows amorphous $\left(V_{N}\right.$ type pit pattern). (E) Cross section of surgically resected specimen shows deep subumucosal invasion with desmoplastic reaction $(H \& E$ stain, $\times 20)$.

Table 2. NBI Magnifying Findings and Histology/Invasion Depth in Hiroshima Classification

\begin{tabular}{|c|c|c|c|c|c|}
\hline \multirow{3}{*}{ NBI magnification findings } & \multirow{3}{*}{ No. of cases } & \multicolumn{4}{|c|}{ Histologic findings } \\
\hline & & \multirow{2}{*}{$\mathrm{HP}$} & \multirow{2}{*}{$\mathrm{TA}$} & \multicolumn{2}{|c|}{ Carcinoma } \\
\hline & & & & M/SM-s & SM-d \\
\hline A & $44(100)$ & $42(95.5)$ & $2(4.5)$ & & \\
\hline B & $287(100)$ & $2(0.7)$ & $221(77.0)$ & $64(22.3)$ & \\
\hline $\mathrm{C} 1$ & $135(100)$ & & $42(31.1)$ & $88(65.2)$ & $5(3.7)$ \\
\hline $\mathrm{C} 2$ & $42(100)$ & & & $16(38.1)$ & $26(61.9)$ \\
\hline $\mathrm{C} 3$ & $62(100)$ & & & $4(6.5)$ & $58(93.5)$ \\
\hline Total & 570 & 44 & 265 & 172 & 89 \\
\hline
\end{tabular}

Values are presented as number (\%).

NBI, narrow band imaging; HP, hyperplastic lesion; TA, tubular adenoma; M/SM-s, carcinoma with intramucosal to scanty submucosal invasion; SM-d, carcinoma with submucosal deep invasion (an invasion depth of $1,000 \mu \mathrm{m}$ or more).

Tumor NBI Interest Group (CTNIG), an organization for international collaboration, and the use of this classification system is gradually increasing. The members of CTNIG include Shinji Tanaka (Japan), Yasushi Sano (Japan), Douglas K. Rex (USA), Roy M. Soetikno (USA), Thierry Ponchon (France), and Brian P. Saunders (UK).

The NICE classification is a simple category classification consisting of types 1 to 3 and is based on three characteristics: 1) lesion color, 2) microvascular architecture (microvessels), and 3) surface pattern. Type 1 is an index for hyperplastic le- sions, type 2 is an index for adenoma or mucosal carcinoma, and type 3 is an index for deep SM invasive carcinoma. As shown in Table 3, type 3 tumors exhibit a high probability of being deep SM invasive carcinomas. The results of a validation study on the NICE classification have already been reported, ${ }^{20,21}$ and clinical research using this classification has been pursued in various countries in the world.

In the NICE classification, a borderline between each type is set for colorectal tumors, which can be distinguished through close observation with a high-resolution video colono- 
Table 3. Each type in NICE Classification and Histology/Invasion Depth in Colorectal Adenoma and Early Carcinoma

\begin{tabular}{lccccc}
\hline & & \multicolumn{2}{c}{ Histologic findings } \\
\cline { 3 - 6 } NICE classification & No. of cases & HP & TA & \multicolumn{2}{c}{ Carcinoma } \\
\cline { 3 - 6 } & & & $2(4.5)$ & M/SM-s & SM-d \\
Type 1 & $44(100)$ & $2(0.4)$ & $263(56.7)$ & $168(36.2)$ & $31(6.7)$ \\
Type 2 & $464(100)$ & & & $4(6.5)$ & $58(93.5)$ \\
Type 3 & $62(100)$ & 44 & 265 & 172 & 89 \\
Total & 570 & &
\end{tabular}

Values are presented as number (\%).

NICE, narrow band imaging international colorectal endoscopic; HP, hyperplastic lesion; TA, tubular adenoma; M/SM-s, carcinoma with intramucosal to scanty submucosal invasion; SM-d, carcinoma with submucosal deep invasion (an invasion depth of 1,000 $\mu \mathrm{m}$ or more).

scope. If possible, colorectal tumors should be diagnosed using magnifying observation. By using Dual Focus system (Olympus Corp., Tokyo, Japan), which is now commercially available, the NICE classification system can be more easily and simply employed.

\section{PROPER USE OF PIT PATTERN DIAGNOSIS AND NBI MAGNIFYING OBSERVATION}

Many deep SM invasive carcinomas can be definitively diagnosed using NBI magnifying observation. Because NBI magnifying observation can be easily performed without the use of a dye, this examination should be conducted initially. If a colorectal tumor cannot be characterized on NBI magnifying observation, a conventional pit pattern diagnosis should be performed using a dye. Further, if a colorectal tumor cannot be characterized using conventional pit pattern methods or if information on invasion depth or the relationship between the carcinoma and the muscular layer is required, endoscopic ultrasonography is recommended.

For intramucosal lesions, a regular surface pattern can be observed on NBI magnifying observation images of protruded-type colorectal adenomas. Almost all protruded-type adenomas can be qualitatively diagnosed using this surface pattern. If qualitative diagnosis can be performed using NBI magnifying observation, pit pattern diagnosis using a dye can be omitted. In NBI magnifying observation images of superficial-type tumors or nongranular, laterally spreading tumors (LST-NG), some lesions are difficult to evaluate because various surface and vascular patterns exist. ${ }^{22}$ Therefore, qualitative diagnosis using only NBI magnifying observation is difficult to perform in many cases. When NBI magnifying observation cannot be used for appropriately evaluating superficialtype tumors or LST-NG, pit pattern diagnosis using a dye, which is the gold standard for magnifying observation, should be performed. ${ }^{23}$

\section{CONCLUSIONS}

This report explains the invasion depth diagnosis of early colorectal carcinoma with superficial or deep SM invasion, with a focus on pit pattern diagnosis and NBI magnifying observation. NBI magnifying observation for colorectal tumors is performed without a dye. Therefore, this simple technique can contribute to improving efficiency and reducing the cost of colonoscopy. We predict that NBI magnifying observation will become widely used. For accurate diagnosis, it is important to use NBI magnifying observation in combination with pit pattern diagnosis after sufficiently understanding the disadvantages of NBI magnifying observation.

For deep SM invasive carcinomas with a lymph node metastasis rate of approximately $1 \%$, the histopathological factors used for extracting these carcinomas have been investigated. Criteria for radical-cure evaluation of SM colorectal carcinoma after endoscopic treatment have been proposed. ${ }^{24} \mathrm{Howev-}$ er, these factors and criteria are still being studied. They will be improved in the near future. On the other hand, endoscopic resection techniques such as endoscopic SM dissection have to be generalized under the enough quality control to prevent incomplete endoscopic treatment, which disturbs the precise histological curative judgment. Currently, however, it seems too early to apply endoscopic treatment such as total excisional biopsy for all deep SM invasive colorectal carcinomas.

\section{Conflicts of Interest}

The authors have no financial conflicts of interest.

\section{REFERENCES}

1. Tanaka S, Terasaki M, Hayashi N, Oka S, Chayama K. Warning for unprincipled colorectal endoscopic submucosal dissection: Accurate diagnosis and reasonable treatment strategy. Dig Endosc 2013;25:107-116.

2. Watanabe T, Itabashi M, Shimada Y, et al. Japanese Society for Cancer of the Colon and Rectum (JSCCR) guidelines 2010 for the treatment of colorectal cancer. Int J Clin Oncol 2012;17:1-29.

3. Kunihiro M, Tanaka S, Haruma K, et al. Electrocautery snare resection stimulates cellular proliferation of residual colorectal tumor: an increasing gene expression related to tumor growth. Dis Colon Rectum 2000; 
43:1107-1115.

4. Kudo S, Tamura S, Nakajima T, Yamano H, Kusaka H, Watanabe H. Diagnosis of colorectal tumorous lesions by magnifying endoscopy. Gastrointest Endosc 1996;44:8-14.

5. Tanaka S, Kaltenbach T, Chayama K, Soetikno R. High-magnification colonoscopy (with videos). Gastrointest Endosc 2006;64:604-613.

6. Sakamoto T, Matsuda T, Matsumoto M, et al. Endoscopic features of poorly differentiated adnocarcinoma in the colorectm. Stomach Intest 2010;45:1750-1756.

7. Kimura T, Yamamoto E, Yamano HO, et al. A novel pit pattern identifies the precursor of colorectal cancer derived from sessile serrated adenoma. Am J Gastroenterol 2012;107:460-469.

8. Yao T, Sugai T, Iwashita A, et al. Histopathological characteristics and diagnostic criteria of SSA/P, from project research "potential of cancerization of colorectal serrated lesions" of the Japanese Society for Cancer of the Colon and Rectum. Stomach Intest 2011;46:442-448.

9. Kanao H, Tanaka S, Oka S, et al. Clinical significance of type V(I) pit pattern subclassification in determining the depth of invasion of colorectal neoplasms. World J Gastroenterol 2008;14:211-217.

10. Masaki T, Katada C, Nakayama M, et al. Narrow band imaging in the diagnosis of intra-epithelial and invasive laryngeal squamous cell carcinoma: a preliminary report of two cases. Auris Nasus Larynx 2009;36: 712-716.

11. Gono K, Obi T, Yamaguchi M, et al. Appearance of enhanced tissue features in narrow-band endoscopic imaging. J Biomed Opt 2004;9: 568-577.

12. Sano Y, Muto M, Tajiri H, Ohtsu A, Yoshida S. Optical/digital chromoendoscopy during colonoscopy using narrow-band imaging system. Dig Endosc 2005;17(Suppl 1):S43-S48.

13. Oba S, Tanaka S, Oka S, et al. Characterization of colorectal tumors using narrow-band imaging magnification: combined diagnosis with both pit pattern and microvessel features. Scand J Gastroenterol 2010; 45:1084-1092.

14. Ikematsu H, Matsuda T, Emura F, et al. Efficacy of capillary pattern type IIIA/IIIB by magnifying narrow band imaging for estimating depth of invasion of early colorectal neoplasms. BMC Gastroenterol 2010;
10:33.

15. Kanao H, Tanaka S, Oka S, Hirata M, Yoshida S, Chayama K. Narrowband imaging magnification predicts the histology and invasion depth of colorectal tumors. Gastrointest Endosc 2009;69(3 Pt 2):631-636.

16. Wada Y, Kudo SE, Kashida H, et al. Diagnosis of colorectal lesions with the magnifying narrow-band imaging system. Gastrointest Endosc 2009; 70:522-531.

17. Tanaka S, Sano Y. Aim to unify the narrow band imaging (NBI) magnifying classification for colorectal tumors: current status in Japan from a summary of the consensus symposium in the 79th Annual Meeting of the Japan Gastroenterological Endoscopy Society. Dig Endosc 2011;23 Suppl 1:131-139.

18. Hirata M, Tanaka S, Oka S, et al. Evaluation of microvessels in colorectal tumors by narrow band imaging magnification. Gastrointest Endosc 2007;66:945-952.

19. Oba S, Tanaka S, Sano Y, Oka S, Chayama K. Current status of narrowband imaging magnifying colonoscopy for colorectal neoplasia in Japan. Digestion 2011;83:167-172.

20. Hewett DG, Kaltenbach T, Sano Y, et al. Validation of a simple classification system for endoscopic diagnosis of small colorectal polyps using narrow-band imaging. Gastroenterology 2012;143:599-607.

21. Nakayama N, Tanaka S, Sano Y, et al. Validation of the narrow band imaging (NBI) international colorectal endoscopic (NICE) classification for prediction of deep submucosal invasive carcinoma. Gastrointest Endosc 2012;75:AB338-AB339.

22. Takata $S$, Tanaka $S$, Hayashi N, et al. Characteristic magnifying narrowband imaging features of colorectal tumors in each growth type. Int J Colorectal Dis. Epub 2012 Dec 4. DOI: http://dx.doi.org/10.1007/ s00384-012-1612-6.

23. Hayashi N, Tanaka S, Kanao H, Oka S, Yoshida S, Chayama K. Relationship between narrow-band imaging magnifying observation and pit pattern diagnosis in colorectal tumors. Digestion 2013;87:53-58.

24. Nakadoi K, Tanaka S, Kanao H, et al. Management of T1 colorectal carcinoma with special reference to criteria for curative endoscopic resection. J Gastroenterol Hepatol 2012;27:1057-1062. 